\title{
Um esquema regenerativo visível em cadeias de alcance variável não limitadas
}

\section{Divanilda Maia Esteves}

\author{
TESE APRESENTADA \\ $\mathrm{AO}$ \\ INSTITUTO DE MATEMÁTICA E ESTATÍSTICA \\ DA \\ UNIVERSIDADE DE SÃO PAULO \\ PARA OBTENÇÃO DO GRAU DE DOUTOR \\ EM \\ CIÊNCIAS

\begin{abstract}
Área de Concentração: Estatística Orientador: Prof. Dr. Antonio Galves
\end{abstract}

Esta tese recebeu apoio financeiro do $\mathrm{CNPq}$

- São Paulo, Março de 2007 - 


\title{
Um esquema regenerativo visível em cadeias de alcance variável não limitadas
}

\author{
Este exemplar corresponde à redação \\ final da tese devidamente corrigida \\ e apresentada por Divanilda Maia Esteves \\ e aprovada pela Comissão Julgadora.
}

São Paulo, março de 2007.

Banca Examinadora:

- Prof. Dr. Pablo Augusto Ferrai (IME-USP)

- Prof. Dr. Miguel Natalio Abadi (IMECC-UNICAMP)

- Prof. Dr. Jesus Enrique Garcia (IMECC-UNICAMP)

- Profa. Dra. Nancy Lopes Garcia (IMECC-UNICAMP)

- Profa. Dra. Chang Chung Yu Dorea (UNb) 
Dedico esta tese ao meu marido, Gustavo, porque sem ele tudo teria sido muito mais difícil. 


\section{Agradecimentos}

Gostaria de agradecer a Deus e ao meu mentor pelas boas inspirações e pela companhia constante.

Agradeço muito ao meu orientador Antonio Galves pela atenção, por todas as coisas que me ensinou e que certamente foram fundamentais para o meu amadurecimento como pesquisadora. Agradeço também ao professor Pablo Ferrari que foi meu orientador de programa e ao professor Francisco Cribari Neto que foi o responsável pela minha vinda à USP. Esses três professores são exemplos de pesquisadores nos quais eu quero me espelhar sempre. Agradeço ainda à professora Sílvia Ferrari por ter nos recebido tão bem quando chegamos em São Paulo.

O doutorado não é simples e muitas vezes eu fiquei desanimada... Por isso eu gostaria de agradecer a todos os amigos que sempre me deram força, incentivo e com os quais eu passei muitos momentos bem felizes: Patrícia e Raydonal, Tatiane e Alessandro, Horácio, Elier, Clodis, Florência, Cristian, Mauricio, Sandro (baiano). Agradeço à Liliam que foi uma grande amiga que fiz neste doutorado e que, entre um café e outro, me ajudou demais. Meu agradecimento especial à Michelli, que está comigo há 13 anos, participando ativamente de toda a minha vida acadêmica e que é a minha grande amiga. Gostaria de agradecer aos meus sogros e meu cunhado (minha nova família), meus pais, meus irmãos, que mesmo de longe sempre vibraram e torceram por mim. 
Enfim, gostaria de agradecer ao Gustavo, não só por sempre saber como resolver meus problemas com o computador, mas principalmente porque é para mim um exemplo de paciência e dedicação. É uma pessoa que sempre estava de bom humor - mesmo quando eu não estava muito legal - e que é o melhor companheiro de caminhada que eu poderia desejar...

Agradeço à banca pelas sugestões.

Agradeço ao CNPq pelo apoio financeiro sem o qual a realização deste trabalho não teria sido possível. 


\section{Resumo}

O objetivo central desta tese é demonstrar a existência de uma estrutura regenerativa visível para cadeias de alcance variável não limitadas. Também apresentamos um algoritmo de identificação de seqüências de instantes de regeneração que converge quase certamente quando o tamanho da amostra diverge. 


\section{Abstract}

Our main aim is prove the existence of a regeneration scheme in unbounded variable length chains. We present an algorithm to identify sequences of regeneration times which converges almost surely as the sample length. 


\section{Sumário}

1 Introdução $\quad 1$

2 Cadeias de Alcance Variável $\quad 3$

2.1 Existência e unicidade de cadeias de alcance variável não limitadas . . . . . . . 7

2.2 Algoritmo Contexto . . . . . . . . . . . . . . . . . 9

2.3 Estrutura regenerativa . . . . . . . . . . . . . . . . . . . 10

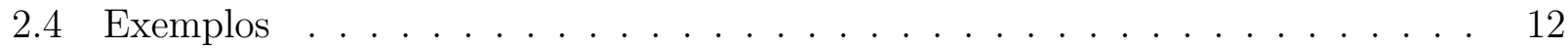

3 Estrutura regenerativa em cadeias de alcance variável $\quad 15$

3.1 Cadeias esparsas . . . . . . . . . . . . . . . . . . . . . . 17

3.2 Caso geral . . . . . . . . . . . . . . . . . . . 23

4 Identificação Empírica da Estrutura Regenerativa 34 


\section{Lista de Figuras}

2.1 Árvore de Contextos referente à cadeia no Exemplo 2.1 . . . . . . . . . . . . . 12

2.2 Árvore de Contextos referente à uma cadeia esparsa . . . . . . . . . . . . . . . 13

3.1 Árvore de Contextos referente à cadeia no Exemplo 3.1 . . . . . . . . . . . 16 


\section{Capítulo 1}

\section{Introdução}

Cadeias de alcance variável foram introduzidas por Rissanen (1983) e popularizadas na literatura estatística por Bühlmann \& Wyner (1999) no caso limitado e no caso não limitado por Ferrari \& Wyner (2003). Recentemente Duarte et al. (2006) deram uma majoração para a velocidade de convergência do algoritmo Contexto para cadeias de alcance variável não limitadas. Nessa classe de modelos, para se decidir sobre o próximo estado da cadeia, em vez de considerarmos todo o passado, consideramos apenas a parte do passado que é relevante, o que foi chamado por Rissanen de contexto. Isso tem duas vantagens óbvias. Primeiramente, os contextos capturam propriedades estruturais dos dados. Em segundo lugar, esses modelos fazem um uso parcimonioso dos parâmetros sem perder a fineza de ajuste.

O objetivo central desta tese é demonstrar a existência de uma estrutura regenerativa visível para as cadeias de alcance variável não limitadas. Através da identificação de tal estrutura, é possível particionar uma dada amostra em blocos independentes e identicamente distribuídos. Essa decomposição em blocos facilita a demonstração de resultados probabilísticos importantes (Lalley 2000) e também pode ser usada para reamostragem. Inicialmente, vamos 
analisar o caso das cadeias esparsas, que como veremos no Capítulo 2, nada mais são que um processo de renovação. Depois vamos ver que qualquer cadeia de alcance variável pode ser representada na forma

$$
\tau=\tau^{[\emptyset]} \cup \bigcup_{k=0}^{\infty} \tau^{[k]}
$$

onde $\tau^{[\emptyset]}$ ou é o conjunto vazio ou tem um único elemento que é uma seqüência de zeros, $\tau^{[k]}=\widetilde{\tau}^{[k]} \times 0^{k}$, com $0^{k}$ sendo uma seqüência de $k$ zeros e $\widetilde{\tau}^{[k]}$ é uma árvore de contextos cuja raiz é o símbolo 1.

Usando tal representação, vamos apresentar condições para que exista um esquema regenerativo nessas cadeias. Por fim, apresentamos um algoritmo de identificação de uma seqüência de instantes de regeneração que converge quase certamente quando o tamanho da cadeia diverge.

No Capítulo 2, vamos fazer uma breve revisão sobre as cadeias de alcance variável. No Capítulo 3, apresentaremos resultados originais referentes à estrutura regenerativa das cadeias de alcance variável não limitadas. No Capítulo 4, descreveremos um algoritmo para encontrar os pontos de regeneração em uma amostra e apresentaremos um resultado referente a convergência do algoritmo. 


\section{Capítulo 2}

\section{Cadeias de Alcance Variável}

As cadeias de Markov, apesar de serem uma boa alternativa para modelar muitos problemas, são, em geral, inapropriadas no que se refere à questão prática da estimação. Uma cadeia de Markov $X_{n}$ de ordem $d$ assumindo valores em um alfabeto $A$ finito tem $|A|^{d}(|A|-1)$ parâmetros a serem estimados (onde $|A|$ representa a cardinalidade de $A$ ). Este número cresce exponencialmente em $d$ e, portanto, para valores não tão grandes de $d$ temos uma quantidade muito grande de parâmetros no modelo. Para termos uma idéia, uma cadeia de ordem 10 sobre um alfabeto com 4 símbolos tem aproximadamente $3.1 \times 10^{6}$ parâmetros a serem estimados (Mächler \& Bühlmann 2002). Uma alternativa mais viável do ponto de vista da estimação, é a utilização de cadeias de Markov de alcance variável (VLMC). Antes de mais nada, vamos relembrar alguns conceitos fundamentais para definirmos tais cadeias.

Por convenção, nos condicionamentos, escreveremos o passado começando com o passado mais recente e indo para o passado mais remoto. Por questão de simplicidade, a seguinte 
notação será utilizada:

$$
\begin{gathered}
x_{m}^{n}:=\left(x_{m}, x_{m+1}, \ldots, x_{n}\right), \text { para } m<n \\
p\left(x_{0} \mid x_{-n}^{-1}\right):=\mathbb{P}\left(X_{0}=x_{0} \mid X_{-1}=x_{-1}, \ldots, X_{-n}=x_{-n}\right), \\
p\left(x_{0} \mid x_{-\infty}^{-1}\right):=\mathbb{P}\left(X_{0}=x_{0} \mid X_{-1}=x_{-1}, X_{-2}=x_{-2}, \ldots\right) .
\end{gathered}
$$

e também

$$
x_{m}^{n} a=x_{m}, \ldots, x_{n}, a
$$

para $a \in A$ e $x_{m}^{n}$ uma seqüência de elementos de $A$, que representa a seqüência obtida ao concatenarmos $x_{m}^{n}$ e $a$, e por fim, vamos representar por $|\omega|$ o comprimento da seqüência $\omega$. Quando apresentamos as seqüências, elas aparecem na ordem natural, com $-\infty$ à esquerda e $\infty$ à direita. Nos condicionamentos, elas são apresentadas do passado mais recente para o mais remoto.

Definição 2.1 Um processo $\left(X_{n}\right)_{n \in \mathbb{Z}}$ assumindo valores em um conjunto $A$ é uma cadeia de Markov de ordem $k$ se o estado presente do processo é influenciado apenas pelos $k$ momentos passados, ou seja

$$
\mathbb{P}\left(X_{0}=x_{0} \mid X_{-\infty}^{-1}=x_{-\infty}^{-1}\right)=p\left(x_{0} \mid x_{-k}^{-1}\right)
$$

Dizemos ainda que uma cadeia de Markov é homogênea no tempo se

$$
p\left(x_{n} \mid x_{n-k}^{n-1}\right)=p\left(x_{0} \mid x_{-k}^{-1}\right)
$$

para qualquer $n \in \mathbb{Z}$.

No que segue, consideraremos apenas cadeias homogêneas no tempo. 
Definição 2.2 Seja $\left(X_{t}\right)_{t \in \mathbb{Z}}$ um processo estacionário com valores em um alfabeto finito $A$ com probabilidades de transição dadas por uma função $P: A \times A^{\{\ldots,-2,-1\}}$ tal que

$$
\mathbb{P}\left(X_{0}=x_{0} \mid X_{-\infty}^{-1}=x_{-\infty}^{-1}\right)=p\left(x_{0} \mid x_{-\infty}^{-1}\right)
$$

para todo $x_{0} \in A, x_{-\infty}^{-1} \in A^{\{\ldots,-2,-1\}}$. Chamamos tal processo de cadeia de ordem infinita com probabilidades de transição $p(\cdot \mid \cdot)$.

No entanto, nem sempre o estado presente depende de todo o passado e neste caso, ao supor que a cadeia é de alcance infinito, estamos considerando mais parâmetros do que realmente precisamos. Assim, podemos definir as cadeias de alcance variável, as quais consideram apenas a parte do passado que é relevante para cada estado presente.

Definição 2.3 A função contexto do processo $\left(X_{n}\right)_{n \in \mathbb{Z}}$, dada por

$$
\begin{aligned}
c: A^{\{\ldots,-2,-1\}} & \rightarrow \underline{A}=\bigcup_{n=1}^{\infty} A^{\{-n, \ldots,-1\}} \\
x_{-\infty}^{-1} & \mapsto x_{-l\left(x_{-\infty}^{-1}\right)}^{-1}
\end{aligned}
$$

onde $l: \underline{A} \rightarrow\{1,2,3, \ldots\}$ é a função comprimento e é dada por

$$
l\left(x_{-\infty}^{-1}\right)=\min \left\{k: p\left(a \mid x_{-\infty}^{-1}\right)=p\left(a \mid x_{-k+1}^{-1}\right), \forall a \in A\right\}
$$

O nome contexto remete ao fato de que a parte do passado da qual depende a variável aleatória $X_{0}$ é uma função da configuração $x_{-\infty}^{-1}$. A função l indica quanto do passado influencia a v.a. $X_{0}$.

Definição 2.4 Seja $k=\sup \left\{l\left(x_{-\infty}^{0}\right): x_{-\infty}^{0} \in A^{\{\ldots,-1,0\}}\right\}$. Se $k<\infty$, então $\left(X_{n}\right)_{n \in \mathbb{Z}}$ é uma cadeia de Markov de alcance variável (VLMC) de ordem $k$. Se a função comprimento 
for não limitada, ou seja, se $k=+\infty$ então temos uma cadeia de alcance variável não limitada.

Informalmente, podemos dizer que nas cadeias de alcance variável, o estado presente da cadeia depende do passado até observarmos um determinado evento que nos faz esquecer o resto do passado. Em outras palavras, o que temos é: considerando, como antes, $l$ como o comprimento de um dado contexto, então $\mathbf{1}_{\{l=k\}}=F\left(X_{-1}^{-k}\right)$, isto é o evento $\{l=k\}$ é um tempo de parada, pois é totalmente explicado por $X_{-1}^{-k}$.

Para mais detalhes sobre as cadeias de ordem infinita, ver Fernández et al. (2001) e sobre cadeias de alcance variável não limitadas não limitadas Duarte et al. (2006).

Se supusermos que a cadeia de Markov de alcance variável é estacionária, sua distribuição pode ser totalmente especificada por suas probabilidades de transição. Sendo assim, pode-se representar o modelo segundo uma árvore de contextos. No caso ilimitado, também podemos usar tal representação, sob certas condições que são discutidas em Duarte et al. (2006). De acordo com a notação acima especificada, uma árvore de contexto completa para uma VLMC $\left(X_{n}\right)_{n \in \mathbb{Z}}$ de alcance $k$ é uma árvore com ramos $\left\{r: r=c\left(x_{-k}^{-1}\right), x_{-k}^{-1} \in A^{\{-k, \ldots,-1\}}\right\}$, que pode ser definida formalmente da seguinte maneira (Csiszár \& Talata 2006).

Definição 2.5 Um subconjunto enumerável $\tau$ de $\bigcup_{k=1}^{\infty} A^{\{-k, \ldots,-1\}}$ é uma árvore completa enraizada com ramos finitos se satisfaz as seguintes condições:

1. Propriedade do sufixo: Para qualquer sequência $\omega_{-k}^{-1} \in \tau$, não existe $u_{-j}^{-1} \in \tau$, com $j<k$, tal que $\omega_{-i}=u_{-i}$, para $i=1, \ldots, j$.

2. Completitude: $\tau$ define uma partição de $A^{\cdots,-2,-1}$. Cada elemento da partição coincide com o conjunto das seqüências em $A^{\cdots,-2,-1}$ que tem $\omega_{-k}^{-1}$ como sufixo. 
Definição 2.6 Uma árvore probabilística de contextos em um alfabeto A é um par ordenado $(\tau, p)$ tal que

- $\tau$ é uma árvore completa

- $p=\{p(\cdot \mid \omega), \omega \in \tau\}$ é uma família de probabilidades de transição em $A$.

Notação: Seja $\tau$ uma árvore de contextos completa. Denotaremos por $c_{\tau}\left(x_{-\infty}^{-1}\right)$ o único sufixo de $x_{-\infty}^{-1}$ que está em $\tau$.

O conjunto $\tau$ pode ser identificado por um conjunto de ramos da seguinte forma:

- o primeiro nó é a raiz, ou o presente;

- os galhos são o passado, sendo que quanto mais longe da raiz, mais distante é o passado;

- cada nó tem no máximo $|A|$ arestas;

- cada contexto é representado por um ramo completo.

\subsection{Existência e unicidade de cadeias de alcance variável}

\section{não limitadas}

Uma cadeia estocástica estacionária $\left(X_{t}\right)$ é compatível com uma árvore probabilística de contextos $(\tau, p)$ se para todo passado infinito $x_{-\infty}^{-1}$ e todo símbolo $a \in A$ tivermos

$$
\mathbb{P}_{p}\left\{X_{0}=a \mid X_{-\infty}^{-1}=x_{-\infty}^{-1}\right\}=p\left(a \mid x_{-\ell}^{-1}\right)
$$

onde $x_{-\ell}^{-1}$ é o único elemento de $\tau$ que é um sufixo do passado $x_{-\infty}^{-1}$. 
No caso em que a árvore de contextos $\tau$ não é finita, a compacidade de $A^{\mathbb{Z}}$ garante que existe ao menos uma cadeia estacionária compatível com $(\tau, p)$. As seguintes condições garantem a unicidade da cadeia.

Definição 2.7 Uma árvore probabilística de contextos $(\tau, p)$ em A é do tipo A se suas probabilidades de transição p satisfazem as seguintes condições.

1. Fracamente não nula, isto é

$$
\sum_{a \in A} \inf _{w \in \tau} p(a \mid w)>0
$$

2. Continuidade, isto é

$$
\beta(k):=\max _{a \in A} \sup \left\{|p(a \mid w)-p(a \mid v)|, v \in \tau, w \in \tau \text { with } w_{-k}^{-1}=v_{-k}^{-1}\right\} \rightarrow 0
$$

quando $k \rightarrow \infty$. definimos também

$$
\beta(0)=\max _{a \in A} \sup \left\{|p(a \mid w)-p(a \mid v)|, v \in \tau, w \in \tau \text { with } w_{-1} \neq v_{-1}\right\}
$$

A seqüência $\{\beta(k)\}_{k} \in \mathbb{N}$ é chamada taxa de continuidade.

Como observado em Duarte et al. (2006), para uma árvore probabilística de contextos do tipo A com uma taxa de continuidade somável, o argumento do acoplamento maximal usado em Fernández \& Galves (2002) implica a unicidade da lei da cadeia consistente com ela.

No nosso teorema geral de existência de um esquema regenerativo, vamos supor que nossas cadeias assumem valores no $\{0,1\}$, são contínuas com taxa de continuidade somável e que além disso, $\inf \{p(1 \mid v): v \in \tau\}=\delta>0$. Isso implica a unicidade da cadeia. 


\subsection{Algoritmo Contexto}

Vamos descrever agora um algoritmo usado para estimar a função contexto a partir de uma amostra $X_{0}, X_{1}, \ldots, X_{n-1}$ de uma VLMC e também as probabilidades de transição associadas a tal cadeia. Esse algoritmo se chama algoritmo Contexto e foi introduzido por Rissanen (1983) no caso limitado e mais tarde popularizado no meio estatístico por Bühlmann \& Wyner (1999). Duarte et al. (2006) apresentam uma majoração da velocidade de convergência desse algoritmo que se aplica também no caso em que $\tau$ é não limitada.

Inicialmente, consideramos candidatos a contextos $X_{n-k(n)}^{n-1}$ onde $k(n)=C_{1} \log n$, sendo que $C_{1}$ é uma constante positiva adequadamente escolhida. A razão intuitiva para escolher o tamanho máximo dos contextos igual a $C_{1} \log n$ é a impossibilidade de estimar seqüências muito maiores que $\log n$ com base numa amostra de tamanho $n$. Em seguida, vamos encurtando (ou podando) esses contextos de acordo com uma seqüência de testes baseados no estatística de razão de verossimilhanças. De maneira mais formal, definimos tal procedimento da seguinte forma.

Seja $X_{0}, X_{1}, \ldots, X_{n-1}$ uma amostra de uma árvore finita $\tau$. Para qualquer seqüência finita $\omega_{-j}^{-1}$, com $j \leq n$, definimos

$$
N_{n}\left(\omega_{-j}^{-1}\right)=\sum_{t=0}^{n-j} \mathbf{1}\left\{X_{t}^{t+j-1}=\omega_{-j}^{-1}\right\}
$$

como sendo o número de ocorrências da seqüência na amostra. Se $N_{n}\left(\omega_{-j}^{-1}\right)>0$, definimos o estimador da probabilidade de transição por

$$
\widehat{p}_{n}\left(a \mid \omega_{-j}^{-1}\right)=\frac{N_{n}\left(\omega_{-j}^{-1} a\right)}{\sum_{b \in A} N_{n}\left(\omega_{-j}^{-1} b\right)},
$$

onde $\left(\omega_{-j}^{-1} a\right)$ denota a seqüência $\left(\omega_{-j}, \ldots, \omega_{-1}, a\right)$, obtido ao concatenarmos $\omega_{-j}^{-1}$ e o símbolo a. Se $N_{n}\left(\omega_{-j}^{-1}\right)=0$, definimos $\widehat{p}_{n}\left(a \mid \omega_{-j}^{-1}\right)=1 /|A|$. 
Vamos definir também

$$
\Delta_{n}(i, \omega)=-2 \sum_{\omega_{i} \in A} \sum_{a \in A} N_{n}\left(\omega_{-i}^{-1} a\right) \log \left[\frac{\widehat{p}\left(a \mid \omega_{-i}^{-1}\right)}{\widehat{p}\left(a \mid \omega_{-i+1}^{-1}\right)}\right]
$$

Note que $\Delta_{n}(i, \omega)$ é a estatística do teste de razão de verossimilhança para testar a consistência de uma amostra ao modelo representado por uma árvore $\tau$ contra a alternativa de que a amostra é consistente com a árvore $\tau^{\prime}$, onde $\tau^{\prime}$ é obtida substituindo o ramo $\omega_{-i}^{-1}$ em $\tau$ pelo ramo $\omega_{-i-1}^{-1}$

Por fim, definimos o comprimento do contexto estimado como

$$
\widehat{l}\left(X_{0}^{n-1}\right)=\max \left\{i=2, \ldots, k(n): \Delta_{n}\left(i, X_{n-k(n)}^{n-1}\right)>C_{2} \log n\right\}
$$

Em Duarte et al. (2006), Bühlmann \& Wyner (1999) e Ferrari \& Wyner (2003) podemos ver algumas propriedades deste algoritmo. Este algoritmo está implementado no ambiente de programação estatística $R$, que pode ser obtida em www.r-project.org, no pacote VLMC (Mächler \& Bühlmann 2002).

\subsection{Estrutura regenerativa}

Definição 2.8 Dados $(\tau, p), \omega \in \tau$ e $x_{-\infty}^{\infty}$ uma seqüência de símbolos de $A$, dizemos que $t \in \mathbb{Z}$ é um ponto de regeneração da seqüência relativamente a $\omega$ se

- $x_{t}^{t+|\omega|-1}=\omega$

- $\forall n \geq t, c_{\tau}\left(x_{-\infty}^{n}\right) \subset x_{t}^{n}$.

Usando essa estrutura de regeneração da cadeia, podemos decompor uma amostra em blocos independentes e identicamente distribuídos. De acordo com Lalley (2000), uma vantagem de 
se decompor uma amostra dessa forma é que obtemos blocos independentes, e assim podemos obter, de maneira simples, versões de resultados importantes, tais como o Teorema Central do Limite e a Lei do Logaritmo Iterado. Esses blocos independentes também podem sem usados para reamostragem (Duarte 2003; Bertail \& Clémençon 2006).

Um caso bem claro de estrutura regenerativa, é quando temos um processo de renovação, o qual é definido da seguinte forma.

Uma seqüência de variáveis aleatórias estritamente crescente $\cdots<T_{-K}<T_{-1}<T_{0}<$ $0 \leq T_{1}<T_{2}<\cdots<T_{K}<\ldots$ é um processo de renovação se satisfaz as seguintes condições:

- As variáveis $\ldots, T_{0}, T_{1}, T_{2}-T_{1}, T_{3}-T_{2}, \ldots$ são independentes entre si.

- As variáveis $T_{k+1}-T_{k}, k \in \mathbb{Z}$, são identicamente distribuídas.

A idéia intuitiva é que as variáveis $\left(T_{k}\right)_{k \in \mathbb{Z}}$ modelam os instantes sucessivos de ocorrência de um determinado fenômeno que se repete, após cada ocorrência, independentemente da história passada (Ferrari \& Galves 1997). Ou seja, cada vez que o fenômeno acontece, o passado e o futuro se tornam independentes.

Numa cadeia de Markov de alcance fixo, um exemplo de processo de renovação, seria a seqüência dos instantes de ocorrência de um determinado estado da cadeia. 


\subsection{Exemplos}

Exemplo 2.1 Considere o alfabeto $A=\{0,1,2,3,4\}$ e a função contexto

$$
c\left(x_{-\infty}^{-1}\right)= \begin{cases}4 & \text { para } x_{-1}=4 \text { e qualquer } x_{-\infty}^{-2} \\ 3 & \text { para } x_{-1}=3 \text { e qualquer } x_{-\infty}^{-2} \\ 3,2 & \text { para } x_{-1}=2, x_{-2}=3 \text { e qualquer } x_{-\infty}^{-3} \\ 1,2 & \text { para } x_{-1}=2, x_{-2}=1 \text { e qualquer } x_{-\infty}^{-3} \\ 1 & \text { para } x_{-1}=1 \text { e qualquer } x_{-\infty}^{-2} \\ 0,0 & \text { para } x_{-1}=0, x_{-2}=0 \text { e qualquer } x_{-\infty}^{-3} \\ 3,0 & \text { para } x_{-1}=0, x_{-2}=3 \text { e qualquer } x_{-\infty}^{-3}\end{cases}
$$

A árvore de contextos representando a cadeia acima pode ser vista na Figura 2.1.

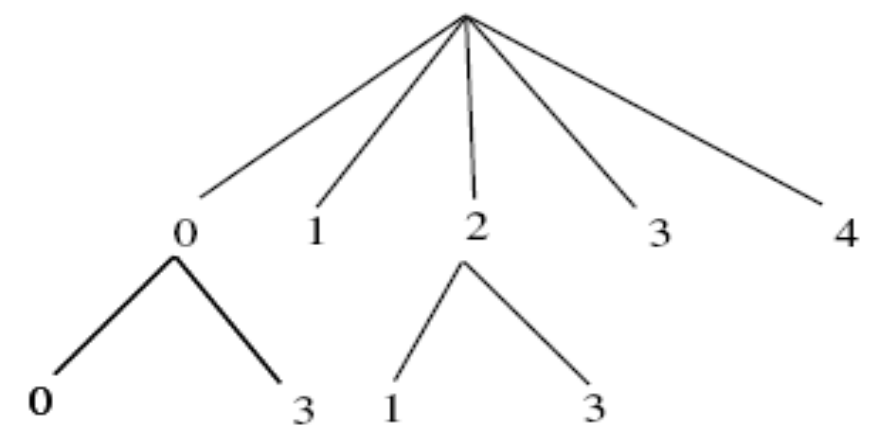

Figura 2.1: Árvore de Contextos referente à cadeia no Exemplo 2.1

Exemplo 2.2 As cadeias de renovação, também chamadas cadeias de alcance variável esparsas, são definidas da seguinte forma (Bühlmann $\&$ Wyner 1999). Dizemos que uma cadeia de alcance variável $\left(X_{n}\right)_{n \in \mathbb{Z}}$ tomando valores no alfabeto $A=\{0,1\}$ é uma cadeia esparsa, se suas probabilidades de transição são dadas por:

$$
p\left(1 \mid x_{-\infty}^{-1}\right)=q_{l\left(x_{-\infty}^{-1}\right)},
$$


onde $l\left(x_{-\infty}^{-1}\right)=l:=\min \left\{i \geq 1: x_{-i}=1\right\}-1$.

Observamos que a cadeia esquece o passado quando encontra um símbolo 1. A Figura 2.2 representa a árvore de contexto da cadeia esparsa.

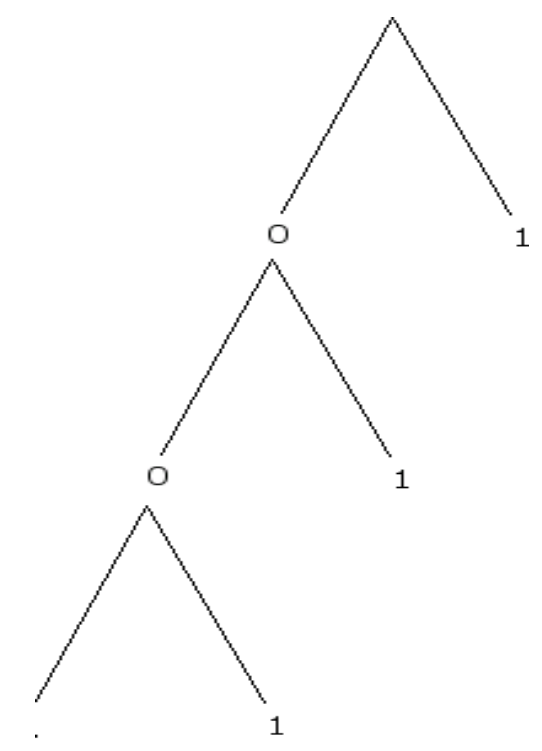

Figura 2.2: Árvore de Contextos referente à uma cadeia esparsa

Observe que as cadeias esparsas nada mais são do que um processo de renovação. Seja $\left(X_{n}\right)_{n \in \mathbb{Z}}$ uma cadeia em um alfabeto finito $\mathcal{A}$ com árvore de contextos $\tau$ e seja $\omega \in \tau$ um ponto de renovação da cadeia. Vamos definir

$$
\mathcal{T}(\omega)=\left\{T_{n}(\omega): n \in \mathbb{Z}\right\}
$$

o conjunto dos pontos em que a cadeia chega ao estado $\omega$.

Seja $\left(Y_{n}\right)_{n \in \mathbb{Z}}$ uma cadeia tal que:

$$
Y_{n}=\left\{\begin{array}{cc}
0 & \text { se } n \in \mathcal{T} \\
1 & \text { se } n \notin \mathcal{T}
\end{array}\right.
$$


E as probabilidades de transição associadas a cadeia seguem a seguinte relação:

$$
\begin{aligned}
& \mathbb{P}\left(T_{2}=m+1 \mid T_{1}=m\right)=\mathbb{P}\left(Y_{1}=1 \mid Y_{0}=1\right)=q_{1} \\
& \mathbb{P}\left(T_{2}=m+2 \mid T_{1}=m\right)=\mathbb{P}\left(Y_{2}=1 \mid Y_{1}=0, Y_{0}=1\right)=\left(1-q_{1}\right) q_{2}
\end{aligned}
$$

e de maneira geral

$\mathbb{P}\left(T_{2}=m+l \mid T_{1}=m\right)=\mathbb{P}\left(Y_{l}=1 \mid Y_{1}=\cdots=Y_{l-1}=0, Y_{0}=1\right)=\left(1-q_{1}\right)\left(1-q_{2}\right) \ldots\left(1-q_{l-1}\right) q_{l}$. 


\section{Capítulo 3}

\section{Estrutura regenerativa em cadeias de}

\section{alcance variável}

Seja $x_{1}, \ldots, x_{n}$ uma amostra da cadeia de alcance variável $\left(X_{n}\right)_{n \in \mathbb{Z}}$ tomando valores no alfabeto finito $A$ associada a uma árvore probabilística $(\tau, p)$. Nosso objetivo é encontrar condições que garantam a existência de uma estrutura regenerativa nessas cadeias.

Vamos começar com um exemplo.

\section{Exemplo 3.1:}

Vamos considerar a cadeia representada pela função contexto

$$
c\left(x_{-\infty}^{-1}\right)= \begin{cases}1 & \text { para } x_{-1}=1 \text { e qualquer } x_{-\infty}^{-2} \\ 1,1,0 & \text { para } x_{-1}=0, x_{-2}=1, x_{-3}=1 \text { e qualquer } x_{-\infty}^{-4} \\ 0,1,0 & \text { para } x_{-1}=0, x_{-2}=1, x_{-3}=0 \text { e qualquer } x_{-\infty}^{-4} \\ 0,0 & \text { para } x_{-1}=0, x_{-2}=0 \text { e qualquer } x_{-\infty}^{-3}\end{cases}
$$

ESta cadeia está representada na Figura 3.1. 


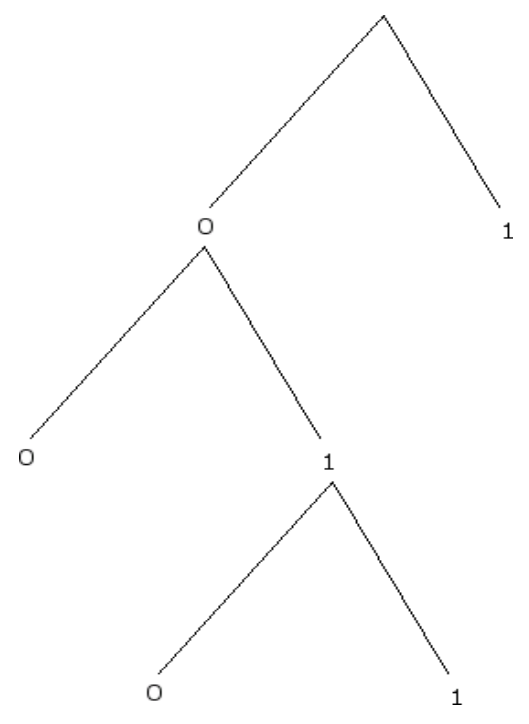

Figura 3.1: Árvore de Contextos referente à cadeia no Exemplo 3.1

Proposição 3.1 Para essa cadeia, se $p(1 \mid 1)>\delta>0$ então existe uma seqüência infinita de instantes

$$
\cdots<\sigma_{0}^{\omega}<0<\sigma_{1}^{\omega}<\sigma_{2}^{\omega}<\ldots
$$

tais que $x_{\sigma_{i}^{\omega}}=x_{\sigma_{i}^{\omega}+1}=1$

Prova: Neste exemplo, é claro que o contexto 1 não será ultrapassado somente se aparecer seguido de 1. Assim, vamos mostrar que a seqüência 11 aparece infinitas vezes.

Vamos definir a seqüência de variáveis aleatórias $\left(Y_{i}\right)_{i \in \mathbb{Z}}$ tal que $Y_{i}=1$ se $X_{i}=X_{i+1}=1 \mathrm{e}$ 0 caso contrário. Desta forma, $\mathbb{P}\left(Y_{i}=1\right)>\delta$. Vamos acoplar $Y_{i}$ com a seqüência de variáveis aleatórias independentes $\left(Z_{i}\right)_{i \in \mathbb{Z}}$, com $\mathbb{P}\left(Z_{i}=1\right)=\delta=1-\mathbb{P}\left(Z_{i}=0\right)$, de modo que $Y_{i} \geq Z_{i}$ para todo $i$. Como

$$
\sum_{i \geq 1} \mathbb{P}\left(Z_{i}=1\right)=\sum_{i \geq 1} \delta=\infty
$$

pelo Lema de Borel-Cantelli, $\mathbb{P}\left(\bigcap_{m \geq 1} \bigcup_{n \geq m}\left\{Z_{i}=1\right\}\right)=1$, e então

$$
\mathbb{P}\left(\bigcap_{m \geq 1} \bigcup_{n \geq m}\left\{Y_{i}=1\right\}\right)=1
$$


o que quer dizer que a seqüência 11 aparece infinitas vezes.

Antes de demonstrar o caso geral das cadeias de alcance variável, vamos apresentar um exemplo com uma cadeia não limitada, que é o caso das cadeias esparsas.

\subsection{Cadeias esparsas}

Numa cadeia esparsa (ver Exemplo 2.2) esquecemos o passado cada vez que encontramos o símbolo 1. Portanto, para obter a estrutura regenerativa, basta garantir, para quase toda amostra suficientemente grande de uma cadeia esparsa, a existência de infinitos uns. Isto é equivalente a procurar as condições necessárias e suficientes para que uma cadeia esparsa cumpra a seguinte igualdade:

$$
\mathbb{P}\left(\sum_{n=1}^{\infty} \mathbf{1}_{\left\{X_{n}=1\right\}}=+\infty \mid X_{0}=1\right)=1
$$

Lema 3.1 Se $\sum_{l=0}^{\infty} q_{l}<1$, então a condição (3.1) nãa se cumpre.

Prova: Vamos mostrar que $\mathbb{P}\left[\bigcap_{n=1}^{+\infty}\left\{X_{n}=0\right\} \mid X_{0}=1\right]>0$, o que quer dizer que a igualdade (3.1) não é verdadeira. Temos que

$$
\mathbb{P}\left[\bigcap_{n=1}^{+\infty}\left\{X_{n}=0\right\} \mid X_{0}=1\right]=1-\mathbb{P}\left[\bigcup_{n=1}^{+\infty}\left\{X_{n}=1\right\} \mid X_{0}=1\right]
$$

Mas

$$
\mathbb{P}\left[\bigcup_{n=1}^{+\infty}\left\{X_{n}=1\right\} \mid X_{0}=1\right]=\sum_{n=1}^{+\infty} \mathbb{P}\left[X_{n}=1, X_{1}^{n-1}=0_{1}^{n-1} \mid X_{0}=1\right]
$$


e para $n \geq 2$

$$
\begin{aligned}
& \mathbb{P}\left[X_{n}=1, X_{1}^{n-1}=0 \mid X_{0}=1\right]= \\
& =\mathbb{P}\left[X_{1}=0 \mid X_{0}=1\right] \times \ldots \times \mathbb{P}\left[X_{n-1}=0 \mid X_{1}^{n-2}=0, X_{0}=1\right] \times \mathbb{P}\left[X_{n}=1 \mid X_{1}^{n-1}=0, X_{0}=1\right] \\
& =\left(1-q_{0}\right) q_{n-1} \prod_{i=2}^{n-1} \mathbb{P}\left[X_{i}=0 \mid X_{1}^{i-1}=0, X_{0}=1\right] \\
& =q_{n-1} \prod_{i=0}^{n-2}\left(1-q_{i}\right)
\end{aligned}
$$

Daí, dado que $\prod_{i=0}^{n-2}\left(1-q_{i}\right)<1$ e $\sum_{l=0}^{\infty} q_{l}<1$, temos que

$$
\begin{aligned}
\mathbb{P}\left[\bigcup_{n=1}^{+\infty}\left\{X_{n}=1\right\} \mid X_{0}=1\right] & =q_{0}+\sum_{n=2}^{+\infty} q_{n-1} \prod_{i=0}^{n-2}\left(1-q_{i}\right) \\
& \leq \sum_{n=0}^{+\infty} q_{n}<1 .
\end{aligned}
$$

Assim, por (3.2) e (3.3), concluímos que a condição (3.1) não é satisfeita.

Teorema 3.1 Se $\sum_{l=0}^{\infty} q_{l}<+\infty$, então a condição (3.1) não se cumpre.

Prova: Se $\sum_{l=0}^{\infty} q_{l}<+\infty$ então existe $\bar{l}$ tal que $\sum_{l>\bar{l}}^{\infty} q_{l}<1$. Temos que

$\mathbb{P}\left[\bigcap_{n=1}^{+\infty}\left\{X_{n}=0\right\} \mid X_{0}=1\right]=\mathbb{P}\left[\bigcap_{n=\bar{l}+1}^{+\infty}\left\{X_{n}=0\right\} \mid X_{0}=1, X_{1}=\cdots=\bar{l}=0\right] \mathbb{P}\left(X_{1}=\cdots=X_{\bar{l}}=0\right)$.

Sabemos que:

- $\mathbb{P}\left(X_{1}=\cdots=\bar{l}=0\right)>0$ 


$$
\begin{aligned}
& \mathbb{P}\left(\bigcap_{n=\bar{l}+1}^{+\infty}\left\{X_{n}=0\right\} \mid X_{0}=1, X_{1}=\cdots=X_{\bar{l}}=0\right) \\
& =1-\mathbb{P}\left(\bigcup_{n=\bar{l}+1}^{+\infty}\left\{X_{n}=0\right\} \mid X_{0}=1, X_{1}=\cdots=X_{\bar{l}}=0\right) \\
& =1-q_{\bar{l}}-\sum_{i=2}^{\infty} \mathbb{P}\left(X_{\bar{l}+i}=1, X_{\bar{l}+1}^{\bar{l}+i-1}=0 \mid X_{0}=1, X_{1}=\cdots=X_{\bar{l}}=0\right) \\
& =1-q_{\bar{l}}-\sum_{i=2}^{\infty} q_{\bar{l}+i-1} \prod_{j=1}^{\bar{l}+i-2}\left(1-q_{j}\right) \\
& \geq 1-\sum_{i=\bar{l}+1}^{\infty} q_{i} \geq 1-\sum_{i=\bar{l}}^{\infty} q_{i}>0
\end{aligned}
$$

Desta forma,

$$
\mathbb{P}\left(\bigcap_{n=1}^{+\infty}\left\{X_{n}=0\right\} \mid X_{0}=1\right)>0,
$$

o que mostra que a igualdade (3.1) não vale neste caso.

Lema 3.2 Vamos supor que $q_{l} \geq \delta>0$, para todo $l=0,1, \ldots$ Nessas condições, podemos construir uma seqüência de variáveis aleatórias independentes e identicamente distribuídas $\left(Y_{n}\right)$ assumindo valores em $\{0,1\}$ acoplada à cadeia $\left(X_{n}\right)$, de tal forma que

- $X_{n} \geq Y_{n}$, para todo $n$ e toda realização das variáveis;

- $\mathbb{P}\left(Y_{n}=1\right)=\delta$.

Prova: Seja $\left(U_{n}\right)$ uma seqüência de variáveis aleatórias independentes e uniformemente distribuídas em $[0,1]$. Para todo $n$, contruímos o par $\left(X_{n}, Y_{n}\right)$ como uma função de $U_{n}$ e de $X_{-\infty}^{n-1}$ da seguinte maneira: 


$$
\left(X_{n}, Y_{n}\right)=F\left(U_{n}, X_{-\infty}^{n-1}\right)=\left\{\begin{array}{l}
(1,1), \text { se } 0 \leq U_{n}<\delta \\
(1,0), \text { se } \delta \leq U_{n}<q_{l}\left(X_{-\infty}^{n-1}\right) \\
(0,0), \text { se } U_{n} \geq q_{l}\left(X_{-\infty}^{n-1}\right)
\end{array}\right.
$$

A seqüência $Y_{n}$ assim definida satisfaz as exigências do lema.

Lema 3.3 Uma condição suficiente para que (3.1) seja válida é que $q_{l}>\delta>0$, para todo $l=0,1, \ldots$

Prova: Seja $\left(Y_{n}\right)_{n \in \mathbb{Z}}$ a seqüência de variáveis aleatórias i.i.d construídas no Lema 3.2. Como

$$
\sum_{n=1}^{+\infty} \mathbb{P}\left(Y_{n}=1\right)=+\infty
$$

pelo lema de Borel-Cantelli, temos

$$
\mathbb{P}\left(\bigcap_{m \geq 1} \bigcup_{n \geq m}\left\{Y_{n}=1\right\}\right)=1
$$

Como

$$
\mathbb{P}\left(\bigcap_{m \geq 1} \bigcup_{n \geq m}\left\{X_{n}=1\right\}\right) \geq \mathbb{P}\left(\bigcap_{m \geq 1} \bigcup_{n \geq m}\left\{Y_{n}=1\right\}\right),
$$

a condição (3.1) é satisfeita. Isso encerra a demonstração do lema.

Teorema 3.2 Seja $\left(X_{n}\right)_{n \in \mathbb{Z}}$ uma cadeia esparsa tal que $\sum_{i=0}^{+\infty} q_{i}=\infty$. Então a igualdade (3.1) é válida.

A prova do teorema 3.2 será feita considerando duas possibilidades complementares para $q_{i}$, a saber, $\lim \sup _{l \rightarrow \infty} q_{l}=\delta>0$ e $\lim \sup _{l \rightarrow \infty} q_{l}=0$. 
Lema 3.4 Se $\limsup _{l \rightarrow \infty} q_{l}=\delta>0$, então a condição (3.1) é válida.

\section{Prova}

Se $\lim \sup _{l \rightarrow \infty} q_{l}=\delta>0$, temos que existem infinitos $l$ tais que $q_{l} \geq \delta$. Vamos chamá-los de $l_{1}, l_{2}, \ldots$, ordenados em forma crescente.

O primeiro bloco, $X_{1}, X_{2}, \ldots, X_{l_{1}}$, pode conter 1 ou não.

Se não contém 1 , a probabilidade de obter $X_{l_{1}+1}=1$ é maior do que $\delta$

$$
\mathbb{P}\left(X_{l_{1}+1}=1 \mid X_{0}=1, X_{1}=\cdots=X_{l_{1}}=0\right)=q_{l_{1}}>\delta .
$$

Por outro lado, se $X_{l_{1}+1}=0$, então vamos considerar o bloco $X_{1}, \ldots, X_{l_{1}}, X_{l_{1}+1}, \ldots, X_{l_{2}}$.

Como antes,

$$
\mathbb{P}\left(X_{l_{2}+1}=1 \mid X_{1}^{l_{2}}=0, X_{0}=1\right)=q_{l_{2}}>\delta
$$

Da mesma forma, concluímos que para todo $i \geq 1$,

$$
\mathbb{P}\left[X_{l_{i}+1}=1 \mid X_{0}=1\right]>\delta
$$

Para concluir, basta acoplar $\left(X_{l_{n}}\right)$ com uma seqüência de variáveis i.i.d de forma análoga à que foi feita no Lema 3.3.

Lema 3.5 Se $\limsup _{l \rightarrow \infty} q_{l}=0$ e $\sum_{l=0}^{\infty} q_{l}=+\infty$, então a condição (3.1) é válida.

Prova: A idéia, usando o procedimento na prova anterior, é mostrar que para todo $0<\delta<1$, existe uma seqüência de instantes $k(n) \rightarrow \infty$ tal que

$$
\mathbb{P}\left[\max \left\{X_{n}, \ldots, X_{n+k(n)}\right\}=1 \mid X_{0}=1\right]>\delta .
$$


E então para provar que (3.1) é válido neste caso, basta acoplar $X_{n}, X_{n+1}, \ldots, X_{n+k(n)}$ e $Y_{n}$, $n=1,2, \ldots$, onde as $\left(Y_{n}\right)_{n \in \mathbb{Z}}$ são variáveis aleatórias i.i.d. com $\mathbb{P}\left(Y_{n}=1\right)=1-\mathbb{P}\left(Y_{n}=0\right)=$ $\delta>0$, de modo que $Y_{n} \leq \max \left\{X_{n}, \ldots, X_{n+k(n)}\right\}$, analogamente ao que foi feito no Lema 3.3. Temos que

$$
\begin{aligned}
& \mathbb{P}\left[\max \left\{X_{n}, \ldots, X_{n+k(n)}\right\}=1 \mid X_{0}=1\right]=1-\mathbb{P}\left[X_{n}^{n+k(n)}=0 \mid X_{0}=1\right] \\
= & 1-\mathbb{P}\left[X_{n+k(n)}=0 \mid X_{n}^{n+k(n)-1}=0, X_{0}=1\right] \\
& \times \mathbb{P}\left[X_{n+k(n)-1}=0 \mid X_{n}^{n+k(n)-2}=0, X_{0}=1\right] \times \ldots \\
& \times \mathbb{P}\left[X_{n+1}=0 \mid X_{n}=0, X_{0}=1\right] \times \mathbb{P}\left[X_{n}=0 \mid X_{0}=1\right] \\
= & 1-\mathbb{P}\left[X_{n}=0 \mid X_{0}=1\right] \prod_{i=1}^{k(n)} \mathbb{P}\left[X_{n+i}=0 \mid X_{n}^{n+i-1}=0, X_{0}=1\right] \\
= & 1-\left(1-\mathbb{P}\left[X_{n}=1 \mid X_{0}=1\right]\right) \prod_{i=1}^{k(n)}\left(1-\mathbb{P}\left[X_{n+i}=1 \mid X_{n}^{n+i-1}=0, X_{0}=1\right]\right) \\
\geq & 1-\left(1-q_{n-1}\right) \prod_{i=1}^{k(n)}\left(1-q_{n+i-1}\right) \\
& \quad k(n) \\
= & 1-\prod_{i=0}\left(1-q_{n+i-1}\right) .
\end{aligned}
$$

Dado que $\sum_{l=0}^{\infty} q_{l}=+\infty$, temos que

$$
\prod_{i=0}^{\infty}\left(1-q_{i}\right) \leq \prod_{i=0}^{\infty} \mathrm{e}^{-q_{i}}=\mathrm{e}^{-\sum_{i=0}^{\infty} q_{i}}=0 .
$$

Logo,

$$
\lim _{k(n) \rightarrow \infty} \prod_{i=1}^{k(n)}\left(1-q_{i}\right)=0 .
$$

Portanto, para todo $0<\delta<1$, existe $n_{0}$ tal que $\forall n>n_{0}$

$$
\mathbb{P}\left[\max \left\{X_{n}, \ldots, X_{n+k(n)}\right\}=1 \mid X_{0}=1\right]>\delta .
$$


Com base nos Teoremas 3.1 e 3.2, concluímos que:

Teorema 3.3 A condição (3.1) é satisfeita se, e somente se, $\sum_{i=0}^{+\infty} q_{i}=\infty$.

\subsection{Caso geral}

Antes de enunciarmos o teorema, precisaremos introduzir uma representação das árvores de contexto.

Proposição 3.2 Qualquer árvore de contextos $\tau$ no alfabeto $\{0,1\}$ pode ser escrita na forma

$$
\tau=\tau^{[\emptyset]} \cup \bigcup_{k=0}^{\infty} \tau^{[k]}
$$

onde

- $\tau^{[\emptyset]}$ ou é o conjunto vazio ou tem um único elemento que é uma seqüência de zeros;

- $\tau^{[k]}=\widetilde{\tau}^{[k]} \times 0^{k}$, com $0^{k}$ sendo uma seqüência de $k$ zeros e $\widetilde{\tau}^{[k]}$ é uma árvore de contextos cuja raiz é o símbolo 1.

Prova: A demonstração da proposição é imediata. Basta reagrupar os contextos de $\tau$ em função do comprimento de seu bloco inicial de 0's.

Notação: Na decomposição proposta acima, $\tilde{\tau}^{[k]}$ é uma árvore com raiz 1. Vamos chamar de $f(k)$ o comprimento máximo dos ramos de $\tilde{\tau}^{[k]}$ excluindo-se a raiz, ou seja

$$
f(k)=\sup \left\{|\omega|-1: \omega \in \tilde{\tau}^{[k]}\right\}
$$

Vamos demonstrar nossos teoremas no caso em que $f(k)<+\infty$, para todo $k \geq 0$, hipótese essa que assumiremos no segue. Vamos inicialmente demonstrar o teorema no caso em que $f$ é 
limitada.

Teorema 3.4 Vamos supor que $(\tau, p)$ seja uma árvore probabilística de contextos no alfabeto $A=\{0,1\}$ satisfazendo as seguintes condições:

- as probabilidades de transição p são contínuas com taxa de continuidade somável;

- $\inf \{p(1 \mid v): v \in \tau\}=\delta>0$

- $f(k) \leq M$, para todo $k \geq 0$, onde $M$ é um inteiro positivo fixado.

Então qualquer que seja $\omega \in \tau$ existem infinitos instantes

$$
\cdots<\sigma_{0}^{\omega} \leq 0<\sigma_{1}^{\omega}<\sigma_{2}^{\omega}<\ldots
$$

que são instantes de regeneração da amostra com relação ao contexto $\omega$.

Observação: As duas primeiras condições garantem a unicidade da cadeia de alcance variável compatível com $(\tau, p)$.

\section{Prova do teorema:}

Para simplificar a redação, vamos supor que $f(0)=0$, isto é, que $\omega=(1)$ é um contexto de $\tau$. Vamos demonstrar o teorema neste caso específico. A adaptação da prova para outro contexto qualquer é imediata.

Como $f(k) \leq M$ para todo $k$, uma condição suficiente para que um instante $n$ seja um ponto de regeneração com relação ao contexto $\omega=(1)$ é que $X_{n}^{n+M-1}=1^{M}$, onde $1^{M}$ é uma seqüência de $M$ 1's. 
Ora,

$$
\mathbb{P}\left(X_{n}^{n+M-1}=1^{M}\right)=\mathbb{P}\left(X_{n}=1\right) p(1 \mid 1)^{M-1} \geq \delta^{M-1} .
$$

Vamos definir

$$
\xi_{n}=\mathbf{1}_{\left\{X_{n}^{n+M-1}=1^{M}\right\}} .
$$

O teorema estará demonstrado se mostrarmos que

$$
\mathbb{P}\left(\bigcap_{m \geq 1} \bigcup_{n \geq m}\left\{\xi_{n}=1\right\}\right)=1
$$

Como as variáveis $\xi_{n}$ não são independentes, não podemos usar o Lema de Borel-Cantelli diretamente. Para resolver o problema, vamos utilizar o seguinte resultado de acoplamento.

Lema 3.6 Podemos construir uma seqüência de variáveis aleatórias independentes e identicamente distribuídas $\left(Y_{n}\right)$ assumindo valores em $\{0,1\}$ acoplada à cadeia $\left(X_{n}\right)$, de tal forma que

- $X_{n} \geq Y_{n}$, para todo $n$ e toda realização das variáveis;

- $\mathbb{P}\left(Y_{n}=1\right)=\delta$.

Prova: Seja $\left(U_{n}\right)$ uma seqüência de variáveis aleatórias independentes e uniformemente distribuídas em $[0,1]$. Para todo $n$, contruímos o par $\left(X_{n}, Y_{n}\right)$ como uma função de $U_{n}$ e de $X_{-\infty}^{n-1}$ da seguinte maneira:

$$
\left(X_{n}, Y_{n}\right)=F\left(U_{n}, X_{-\infty}^{n-1}\right)=\left\{\begin{array}{l}
(1,1), \text { se } 0 \leq U_{n}<\delta \\
(1,0), \text { se } \delta \leq U_{n}<p\left(1 \mid X_{-\infty}^{n-1}\right) \\
(0,0), \text { se } U_{n} \geq p\left(1 \mid X_{-\infty}^{n-1}\right) .
\end{array}\right.
$$

A seqüência $Y_{n}$ assim definida satisfaz as exigências do lema. 
Para concluir a demonstração do teorema definimos

$$
\zeta_{n}=\mathbf{1}_{\left\{Y_{n}^{n+M-1}=1^{M}\right\}}
$$

Pelo Lema 3.6, $\zeta_{n} \leq \xi_{n}$, para todo $n$ e toda realização das variáveis. Portanto

$$
\begin{aligned}
\mathbb{P}\left(\bigcap_{m \geq 1} \bigcup_{n \geq m}\left\{\xi_{n}=1\right\}\right) & \geq \mathbb{P}\left(\bigcap_{m \geq 1} \bigcup_{n \geq m}\left\{\zeta_{n}=1\right\}\right) \\
& \geq \mathbb{P}\left(\bigcap_{m \geq 1} \bigcup_{n \geq 1}\left\{\zeta_{n M}=1\right\}\right)
\end{aligned}
$$

Como as variáveis aleatórias $\zeta_{n M}$ são independentes e identicamente distribuídas, o Lema de Borel-Cantelli garante que

$$
\mathbb{P}\left(\bigcap_{m \geq 1} \bigcup_{n \geq m}\left\{\zeta_{n M}=1\right\}\right)=1
$$

Isso conclui a demonstração do teorema.

Observação: O acoplamento utilizado no lema é muitas vezes chamada de acoplamento maximal (Fernández et al. 2001).

Vamos agora considerar o caso em $f(k)<\infty$ para todo $k$, mas a função $f$ é ilimitada. Vamos definir

$$
\{f=m\}=\{k \in \mathbb{N}: f(k)=m\}
$$

e

$$
a_{n}=\inf \{k \in\{f=n\}\}
$$

Teorema 3.5 Vamos supor que $(\tau, p)$ seja uma árvore de contextos no alfabeto $A=\{0,1\}$ satisfazendo as seguintes condições: 
- as probabilidades de transição p são contínuas com taxa de continuidade somável;

- $\inf \{p(1 \mid v): v \in \tau\}=\delta>0$

- $f(k) \leq f\left(k^{\prime}\right)$, se $k \leq k^{\prime}$ e $f(k) \rightarrow+\infty$, quando $k \rightarrow+\infty$;

- $\sum_{i=1}^{\infty}|\{f=i\}|(1-\delta)^{a_{i}}<\infty$.

Então qualquer que seja $\omega \in \tau$ existem infinitos instantes

$$
\cdots<\sigma_{0}^{\omega} \leq 0<\sigma_{1}^{\omega}<\sigma_{2}^{\omega}<\ldots
$$

que são instantes de regeneração da amostra com relação ao contexto $\omega$.

Observação: As duas primeiras condições garantem a unicidade da cadeia de alcance variável compatível com $(\tau, p)$.

\section{Prova do teorema:}

Para simplificar a redação, vamos supor que $f(0)=0$, isto é, que $\omega=(1)$ é um contexto de $\tau$. Vamos demonstrar o teorema neste caso específico. A adaptação da prova para outro contexto qualquer é imediata.

Podemos supor, sem perda de generalidade, que

$$
\widetilde{\tau}^{[k]}=A^{f(k)} \times\{1\} .
$$

Para toda seqüência $\left(X_{n}\right)$, com $X_{m}=1$ e, para todo $i \geq 1$, vamos definir $D_{i}^{m}$ como sendo o número de 0's sucessivos que devem aparecer a partir do instante $i$, para que tenhamos um contexto que ultrapasse o instante $m$. Formalmente

$$
D_{i}^{m}=\min \left\{j \geq 1:\left|c_{\tau}\left(X_{-\infty}^{m-1} 1 X_{m}^{m+i} 0^{j}\right)\right|>j+i+1\right\}
$$


Lema 3.7 Para toda seqüência $X_{-\infty}^{\infty}$ tal que $X_{m}=1$, temos que

$$
\left\{\bigcap_{i=0}^{\infty}\left|c_{\tau}\left(X_{-\infty}^{m+i}\right)\right|<i\right\}=\bigcap_{i=0}^{\infty}\left\{D_{i}^{m} \geq 1\right\}
$$

Prova: Segue diretamente da definição das variáveis $D_{i}^{m}$.

Fixado $n$, a seqüência $D_{i}^{n}, i \geq 1$, depende da seqüência de passados $X_{-\infty}^{n+i}$. Ou seja, ela tem uma relação de dependência do passado complicada. Para estudar seu comportamento, vamos acoplá-la a uma cadeia de Markov não homogênea $\tilde{D}_{i}^{n}$, assumindo valores em $\mathbb{N}$, cuja matriz de transição $q_{i}\left(d^{\prime} \mid d\right)=\mathbb{P}\left(\tilde{D}_{i}^{n}=d^{\prime} \mid \tilde{D}_{i-1}^{n}=d\right)$, é assim definida

$$
\left\{\begin{array}{l}
q_{i}(0 \mid 0) \quad=1 \\
q_{i}(d-1 \mid d)=1-\delta, \quad d \geq 1 \\
q_{i}\left(a_{i+1} \mid d\right)=\delta, \quad d \geq 1,
\end{array}\right.
$$

onde $a_{i}=\inf \{k \in\{f=i\}\}$.

Lema 3.8 Para todo $n$ e para toda amostra $X_{-\infty}^{\infty}$ tal que $X_{n}=1$, existe um acoplamento entre $D_{i}^{n}$ e $\tilde{D}_{i}^{n}$ de tal forma que $D_{0}^{n}=\tilde{D}_{0}^{n}=1$ e $D_{i}^{n} \geq \tilde{D}_{i}^{n}, \forall i \geq 1$.

Prova: Observamos que, para todo $n$,

$$
D_{i}^{n}=g_{i}\left(D_{i-1}^{n}, X_{n+i}\right)
$$

onde $g_{i}$ é assim definida:

$$
g_{i}(d, x)= \begin{cases}0 & \text { se } d=0 \\ d-1 & \text { se } d \geq 1 \text { e } x=0 \\ a_{i+1} & \text { se } d \geq 1 \text { e } x=1 .\end{cases}
$$

Para cada $n$, seja $\left\{U_{i}^{n}: n \in \mathbb{Z}, i \geq 1\right\}$ uma família de variáveis aleatórias independentes entre si e uniformemente distribuídas em $[0,1]$. Como no Lema 3.6, para cada $n \in \mathbb{Z}$ e para 
cada $i \geq 1$, definimos a variável $\left(Y_{i}^{n}\right)$ assumindo valores em $\{0,1\}$ e acoplada à variável $\left(X_{n+i}\right)$, da seguinte maneira:

$$
\left(X_{n+i}, Y_{i}^{n}\right)=F\left(U_{i}^{n}, X_{-\infty}^{n+i-1}\right)=\left\{\begin{array}{l}
(1,1), \text { se } 0 \leq U_{i}^{n}<\delta \\
(1,0), \text { se } \delta \leq U_{i}^{n}<p\left(1 \mid X_{-\infty}^{n+i-1}\right) \\
(0,0), \text { se } U_{n} \geq X_{-\infty}^{n=i-1}
\end{array}\right.
$$

Observamos que, por construção,

- $X_{n+i} \geq Y_{i}^{n}$.

- $\mathbb{P}\left(Y_{i}^{n}=1\right)=\delta$

- A família $\left\{Y_{i}^{n}: n \in \mathbb{Z}, i \geq 1\right\}$ é independente.

Vamos definir agora

$$
\tilde{D}_{i}^{n}=g_{i}\left(\tilde{D}_{i-1}^{n}, Y_{i}^{n}\right)
$$

onde $g_{i}$ é a função 3.5.

Desta maneira, para todo $n, i$ e para toda realização das variáveis, temos que $\tilde{D}_{i}^{n} \leq D_{i}^{n}$. Isso encerra a demonstração do lema.

Vamos definir as variáveis

$$
\xi_{n}=\mathbf{1}_{\left\{\cap_{i=1}^{\infty}\left\{D_{i}^{n} \geq 1\right\}\right\}}
$$

e

$$
\tilde{\xi}_{n}=\mathbf{1}_{\left\{\cap_{i=1}^{\infty}\left\{\tilde{D}_{i}^{n} \geq 1\right\}\right\}}
$$

Lema 3.9 Nas condições do teorema, temos que $\tilde{\xi}_{n} \leq \xi_{n}$, para todo $n$ e para toda realização das variáveis. 
Prova: Segue diretamente da definição das variáveis $\tilde{\xi}_{n}$ e $\xi_{n}$, usando o Lema 3.8.

Lema 3.10 Nas condições do teorema, temos que

$$
\mathbb{P}\left(\tilde{\xi}_{n}=1\right)>0
$$

\section{Prova:}

Para cada $n$, seja a seqüência de variáveis $\left(\eta_{i}^{n}\right)$ dada por

$$
\begin{aligned}
\eta_{0}^{n} & =1 \\
\eta_{1}^{n} & =\inf \left\{i>1: \tilde{D}_{i}^{n}=a_{i+1}\right\} \\
& \vdots \\
\eta_{i}^{n} & =\inf \left\{i>\eta_{i-1}^{n}: \tilde{D}_{i}^{n}=a_{i+1}\right\}
\end{aligned}
$$

e também a seqüência de variáveis aleatórias

$$
\kappa_{i}^{n}=\eta_{i+1}^{n}-\eta_{i}^{n}-1
$$

As v.a. $\left(\kappa_{i}^{n}\right)$ são i.i.d com distribuição geométrica com parâmetro $\delta$ e

$$
\left\{\tilde{\xi}_{n}=1\right\}=\left\{\kappa_{0}^{n}<\eta_{0}^{n}, \kappa_{1}^{n}<\eta_{1}^{n}, \ldots\right\}
$$

Assim

$$
\mathbb{P}\left(\tilde{\xi}_{n}=1\right)=\mathbb{P}\left(\bigcap_{i=0}^{\infty}\left\{\kappa_{i}^{n} \leq a\left(\eta_{i}^{n}\right)\right\}\right)
$$

Dado que as v.a. $\kappa_{i}^{n}$ são independentes, temos que

$$
\begin{aligned}
\mathbb{P}\left(\tilde{\xi}_{n}=1\right) & =\prod_{i=0}^{\infty} \mathbb{P}\left(\kappa_{i}^{n} \leq a\left(\eta_{i}^{n}\right)\right) \\
& \geq \prod_{i=0}^{M} \mathbb{P}\left(\kappa_{i}^{n}=0\right) \prod_{i=M}^{\infty} \mathbb{P}\left(\kappa_{i}^{n} \leq a\left(\eta_{i}^{n}\right)\right)
\end{aligned}
$$


para todo $M \geq 1$.

Usando o fato de que $\prod_{i}\left(1-a_{i}\right) \geq 1-\sum_{i} a_{i}$, para $a_{i}<1$, o lado direito dessa última desigualdade pode ser reescrito como

$$
\delta^{M} \prod_{i=M}^{\infty}\left(1-(1-\delta)^{a\left(\eta_{i}^{n}\right)}\right) \geq \delta^{M}\left(1-\sum_{i=M}^{\infty}|\{f=i\}|(1-\delta)^{a_{i}}\right)
$$

Como, por hipótese,

$$
\sum_{i=0}^{\infty}|\{f=i\}|(1-\delta)^{a_{i}}<\infty
$$

podemos tomar $M$ suficientemente grande para que,

$$
\sum_{i=M}^{\infty}|\{f=i\}|(1-\delta)^{a_{i}}<1
$$

Daí segue

$$
\mathbb{P}\left(\tilde{\xi}_{n}=1\right)>\delta^{M}\left(1-\sum_{i=M}^{\infty}|\{f=i\}|(1-\delta)^{a_{i}}\right)>0 .
$$

Isso concluímos a demonstração do lema.

Para concluir a demonstração do teorema, precisamos mostrar que

$$
\mathbb{P}\left(\bigcap_{m \geq 1} \bigcup_{n \geq m}\left\{\xi_{n}=1\right\}\right)=1
$$

Pelo Lema 3.9, temos que

$$
\mathbb{P}\left(\bigcap_{m \geq 1} \bigcup_{n \geq m}\left\{\xi_{n}=1\right\}\right) \geq \mathbb{P}\left(\bigcap_{m \geq 1} \bigcup_{n \geq m}\left\{\tilde{\xi}_{n}=1\right\}\right)
$$

A seqüência de variáveis aleatórias $\left(\tilde{\xi}_{n}\right)$ é, por construção, independente, já que as seqüências $\left(U_{i}^{n}\right)_{i \geq 1}$ usadas na sua construção são independentes. Além disso, pelo Lema 3.10

$$
\mathbb{P}\left(\tilde{\xi}_{n}=1\right)>\delta^{M}\left(1-\sum_{i=M}^{\infty}|\{f=i\}|(1-\delta)^{a_{i}}\right)>0
$$


Portanto, pelo Lema de Borel-Cantelli,

$$
\mathbb{P}\left(\bigcap_{m \geq 1} \bigcup_{n \geq m}\left\{\tilde{\xi}_{n}=1\right\}\right)=1
$$

Isso encerra a demonstração do teorema.

Corolário 3.1 No Teorema 3.5, se a função $f$ for invertível, então $\sum_{i=1}^{\infty}|\{f=i\}|(1-\delta)^{a_{i}}<$ $+\infty$ é equivalente a $\sum_{i=1}^{\infty}(1-\delta)^{\left[f^{-1}(i)\right]}<+\infty$.

Prova: segue do fato de que se $f$ é invertível, $a_{k}=f^{-1}(k)$.

Corolário 3.2 No Teorema 3.5, se a $f(k)=k$, então $\sum_{i=1}^{\infty}|\{f=i\}|(1-\delta)^{a_{i}}<+\infty$ é equivalente a $\sum_{i=1}^{\infty}(1-\delta)^{\left[f^{-1}(i)\right]}<+\infty$.

Prova: segue do fato de que $f^{-1}(k)=k$.

A seguir vejamos o que acontece para algumas funções conhecidas. Já vimos no Corolário 3.2 que se $f(k)=k$ o teorema é válido.

Exemplo 3.1 Vamos considerar $f(k)=\ln (k)$. Neste caso, $f^{-1}(k)=\exp (k)$, de onde segue que

$$
\sum_{i=1}^{\infty}(1-\delta)^{f^{-1}(i)} \leq \sum_{i=1}^{\infty}(1-\delta)^{i}<\infty
$$

Então, a condição do Teorema é válida. De maneira mais geral, para qualquer função $f$ tal que $f^{-1}(k)>k, \forall k$, usando o mesmo argumento que foi utilizado no caso $f(k)=\log (k)$, concluímos que $\sum_{i=1}^{\infty}(1-\delta)^{f^{-1}(i)}$ é convergente. 
Exemplo 3.2 Agora vamos considerar $f(k)=\exp (k)$. Queremos mostrar que $\sum_{i=1}^{\infty}(1-\delta)^{\ln (i)}$ é convergente. Sabemos que uma série $\sum_{i=1}^{\infty} a_{n}$ é convergente se e somente se $\sum_{i=1}^{\infty} 2^{k} a_{2^{k}}$ também é (Khuri 2003). Usando esse resultado, vemos que a série é convergente desde que $\delta>1-\frac{1}{2 \ln 2}$. 


\section{Capítulo 4}

\section{Identificação Empírica da Estrutura}

\section{Regenerativa}

No capítulo anterior, demos condições para que existam os pontos de separação propostos no capítulo anterior. Agora, vamos descrever um algoritmo para encontrar tais pontos de separação em uma amostra observada. Em seguida, observaremos o comportamento dos pontos de regeneração que foram identificados em uma dada amostra à medida que aumentamos o tamanho da mesma. Quando a árvore de contextos associada à amostra não for conhecida, usaremos o algoritmo Contexto para encontrar a árvore que melhor modela a amostra. Como foi dito no Capítulo 2, tal algoritmo está implementado na plataforma R, no pacote VLMC. Para maiores informações Mächler \& Bühlmann (2002).

Inicialmente, vamos considerar o caso em que temos um amostra $X_{1}, \ldots, X_{n}$ de $(\tau, p)$, com $\tau$ conhecida. Seja $M_{n}$ o número de pontos de regeneração nesta cadeia. Vamos descrever um algoritmo para encontrar pontos $t_{1}\left(X_{1}^{n}, \tau\right), \ldots, t_{M_{n}}\left(X_{1}^{n}, \tau\right)$ que satisfaçam as condições dos teoremas vistos no capítulo anterior, para um determinado contexto $\omega$. Sejam $\mathcal{S}_{n}=$ 
$\left\{t_{i}\left(X_{1}^{n}, \tau\right) ; 0 \leq i \leq M_{n}\right\}$ o conjunto dos pontos de regeneração em uma amostra $X_{1}, \ldots, X_{n}$ e $\mathcal{S}=\left\{t_{i}\left(X_{1}^{\infty}, \tau\right) ; i \geq 1\right\}$ o conjunto dos pontos de regeneração em $X_{1}, X_{2}, \ldots$

\section{Algoritmo}

Passo 0 - Fixe $\omega \in \tau$. Faça $\mathrm{k}=0$ e $\mathrm{m}=1$.

Passo 1 - Fixe $i_{1}^{(0)}=0$. Se $C\left(X_{1}^{n-i_{1}^{(0)}}\right)=C\left(X_{1}^{n}\right)=\omega$, faça $i_{1}=0$ e $\widetilde{t}_{1}\left(x_{1}^{n}, \tau\right)=n$ e vá ao passo 5.

Passo 2 - Faça $\mathrm{k}=\mathrm{k}+1$. Se $i_{1}^{(k-1)}<n-1$, faça $i_{1}^{(k)}=\min \left\{i_{1}^{(k-1)}<i<n: C\left(X_{1}^{n-i}\right)=\omega\right\}$. Se não existir tal $i_{1}^{(k)}$, faça $\mathcal{S}=\emptyset$ e pare.

Passo 3 - Se $l\left(X_{1}^{n-j}\right)>i_{1}^{(k)}-j+|\omega|$ para algum $0 \leq j<i_{1}^{(k)}$, volte ao passo 2.

Passo 4 - Faça $i_{1}=i_{1}^{(k)}$ e $\widetilde{t}_{1}\left(X_{1}^{n}, \tau\right)=n-i_{1}-|\omega|+1$ e vá ao passo 5 .

Passo 5 - Faça $\mathrm{m}=\mathrm{m}+1$ e $\mathrm{k}=0$. Faça $i_{m}^{(0)}=i_{m-1}-|\omega|$. Se $C\left(X_{1}^{n-i_{m}^{0}}\right)=\omega$, faça $i_{m}=i_{m}^{(0)}$, $\widetilde{t}_{m}\left(x_{1}^{n}, \tau\right)=n-i_{m}-|\omega|+1$ e repita este passo.

Passo 6 - Faça $\mathrm{k}=\mathrm{k}+1$. Se $i_{m}^{(k-1)}<n-1$, faça $i_{m}^{(k)}=\min \left\{i_{m}^{(k-1)}<i<n: C\left(X_{1}^{n-i}\right)=\omega\right\}$. Se não existir tal $i_{m}^{(k)}$, faça $m-1=M_{n}$ e vá ao passo 9 .

Passo 7 - Se $l\left(X_{1}^{n-j}\right)>i_{m}^{(k)}-j+|\omega|$ para algum $i_{m-1} \leq j<i_{m}^{k}$, volte ao passo 6 .

Passo 8 - Faça $i_{m}=i_{m}^{(k)}$ e $\widetilde{t}_{m}\left(X_{1}^{n}, \tau\right)=n-i_{m}-|\omega|+1$. Se $i_{m}<n-1$, volte ao passo 5 .

Passo 9 - Para finalizar, para $i=1, \ldots, M_{n}$, faça $\widetilde{t}_{i}\left(X_{1}^{n}, \tau\right)=t_{M_{n}-i+1}$ e então teremos $\mathcal{S}$.

Não vamos considerar todos os pontos encontrados. Na verdade, vamos considerar os pontos que estão em $X_{1}, \ldots, X_{n-k(n)}$, sendo que $k(n)$ é uma função que cresce com o tamanho da amostra e que $n>k(n)$. Isso porque com probabilidade alta os pontos "próximos" a $n$ não serão pontos de regeneração quando aumentarmos um pouco a amostra.

Com base no algoritmo acima, temos o seguinte resultado, onde encontramos um limite 
superior para a probabilidade de que um determinado ponto de separação (encontrado usando o algoritmo) não esteja em $\mathcal{S}$. Inicialmente vamos demonstrar o resultado para o caso das cadeias esparsas e depois para o outro exemplo.

Teorema 4.1 Seja $X_{1}, \ldots, X_{n}$ uma amostra de uma cadeia esparsa. Vamos denotar os pontos de separação associados a esta amostra por $t_{1}\left(X_{1}^{n}, \tau\right), \ldots, t_{M_{n}}\left(X_{1}^{n}, \tau\right)$. Então, se $\sum_{i=0}^{\infty} q_{i}=\infty$, então

$$
\mathbb{P}\left(\bigcup_{i=1}^{M_{n-k(n)}} t_{i}\left(X_{1}^{n}, \tau\right) \notin \mathcal{S}\right) \leq(n-k(n)) \sum_{z=1}^{\infty} z e^{-\sum_{m=0}^{k(n)+z} q_{m}} \rightarrow 0
$$

sendo que aqui $k(n)$ é tal que $e^{-\sum_{m=0}^{k(n)} q_{m}}=o\left(\frac{1}{n-k(n)}\right)$.

\section{Prova:}

Para simplificar a notação, vamos considerar $t_{i}\left(X_{1}^{n}, \tau\right)=t_{i}$.

Para cada $i$, temos que $\left\{t_{i} \in \mathcal{S}_{n}\right\}=\left\{\left|C_{\tau}\left(X_{0}^{j}\right)\right| \leq j-i: t_{i}+|\omega|-1 \leq j \leq n\right\}$ e $\left\{t_{i} \notin \mathcal{S}\right\}=$ $\left\{\left|C_{\tau}\left(X_{0}^{j}\right)\right|>j-i\right.$ para algum $\left.j \geq t_{i}+|\omega|-1\right\}$. Daí

$$
\begin{aligned}
\mathbb{P}\left(\bigcup_{i=1}^{M_{n-k(n)}} t_{i} \notin \mathcal{S}\right) & =\mathbb{P}\left[\bigcup_{i=1}^{M_{n-k(n)}}\left\{\bigcap_{s=t_{i}+|\omega|-1}^{n}\left\{l\left(X_{1}^{s}\right) \leq s-t_{i}\right\} \cap \bigcup_{j=n+1}^{\infty}\left\{l\left(X_{1}^{j}\right)>j-t_{i}\right\}\right\}\right] \\
& \leq \sum_{i=1}^{M_{n-k(n)}} \mathbb{P}\left[\bigcap_{s=t_{i}+|\omega|-1}^{n}\left\{l\left(X_{1}^{s}\right) \leq s-t_{i}\right\} \cap \bigcup_{j=n+1}^{\infty}\left\{l\left(X_{1}^{j}\right)>j-t_{i}\right\}\right] \\
& \leq \sum_{i=1}^{M_{n-k(n)}} \mathbb{P}\left[\bigcup_{j=n+1}^{\infty}\left\{l\left(X_{1}^{j}\right)>j-t_{i}\right\}\right] \\
& \leq \sum_{i=1}^{M_{n-k(n)}} \mathbb{P}\left[\bigcup_{j=n+1}^{\infty}\left\{l\left(X_{1}^{j}\right)>j-(n-k(n))\right\}\right] \\
& \leq(n-k(n)) \sum_{j=n+1}^{\infty} \mathbb{P}\left(l\left(X_{1}^{j}\right)>j-(n-k(n))\right)
\end{aligned}
$$

pois $\forall i=1, \cdots, M_{n-k(n)}, t_{i} \leq n-k(n)$, o que implica $j-t_{i} \geq j-(n-k(n))$. 
Mas sabemos que

$$
\begin{aligned}
\mathbb{P}\left(l\left(X_{1}^{j}\right)>j-(n-k(n))\right) & =\mathbb{P}\left(X_{j}=\cdots=X_{n-k(n)}=0\right) \\
& =\mathbb{P}\left(X_{0}=\cdots=X_{j-(n-k(n))}=0\right) \\
& =\sum_{i=j-(n-k(n))}^{\infty} \mathbb{P}\left(X_{0}=1, X_{1}=\cdots=X_{i}=0\right) \\
& =p(1) \sum_{i=j-(n-k(n))}^{\infty} \prod_{m=0}^{i-1}\left(1-q_{m}\right) \\
& \leq p(1) \sum_{i=j-(n-k(n))}^{\infty} \exp ^{-\sum_{m=0}^{i-1} q_{m}} .
\end{aligned}
$$

Portanto,

$$
\begin{aligned}
\mathbb{P}\left(\bigcup_{i=1}^{M_{n-k(n)}} t_{i} \notin \mathcal{S}\right) & \leq(n-k(n)) \sum_{j=n+1}^{\infty} p(1) \sum_{i=j-(n-k(n))}^{\infty} \exp ^{-\sum_{m=0}^{i-1} q_{m}} \\
& =(n-k(n)) \sum_{z=1}^{\infty} z e^{-\sum_{m=0}^{k(n)+z} q_{m}} \rightarrow 0,
\end{aligned}
$$

quando $n \rightarrow \infty$, desde que $e^{-\sum_{m=0}^{k(n)} q_{m}}=o\left(\frac{1}{n-k(n)}\right)$.

Teorema 4.2 Dados $X_{1}, \ldots, X_{n}$ uma amostra de $(\tau, p)$ e $t_{1}\left(X_{1}^{n}, \tau\right), \ldots, t_{M_{n}}\left(X_{1}^{n}, \tau\right)$ os pontos de separação associados a esta amostra. Então, sob as hipóteses do Teorema 3.5,

$$
\begin{aligned}
\mathbb{P}\left(\bigcup_{i=1}^{M_{n-k(n)}} t_{i}\left(X_{1}^{n}, \tau\right) \notin \mathcal{S}\right) & \leq(n-k(n)) P(1)\left(\frac{1}{1-2(1-\delta)} \sum_{j=n+1}^{\infty}(2(1-\delta))^{m_{j}}+\sum_{j=1}^{\infty} j(2(1-\delta))^{f\left(m_{n+j}\right)}\right) \\
& \rightarrow 0,
\end{aligned}
$$

onde $k(n)$ é tal que $2(1-\delta))^{f\left(m_{n}\right)}=o\left(\frac{1}{n-k(n)}\right)$ e $\sum_{j=n+1}^{\infty}(2(1-\delta))^{m_{j}}=o\left(\frac{1}{n-k(n)}\right)$ e $m_{j}=$ $\min \{m: m+1+f(m)>j-(n-k(n))\}$.

\section{Prova:}

Da mesma forma que fizemos no dois teoremas anteriores, vamos considerar $t_{i}\left(X_{1}^{n}, \tau\right)=t_{i}$. 
Seguindo os passos da demonstração anterior, temos que

$$
\mathbb{P}\left(\bigcup_{i=1}^{M_{n-k(n)}} t_{i} \notin \mathcal{S}\right) \leq(n-k(n)) \sum_{j=n+1}^{\infty} \mathbb{P}\left(l\left(X_{1}^{j}\right)>j-(n-k(n))\right) .
$$

Mas sabemos que, fazendo $m_{j}=\min \{m: m+1+f(m)>j-(n-k(n))\}$

$$
\begin{aligned}
\mathbb{P}\left(l\left(X_{1}^{j}\right)>j-(n-k(n))\right) & =\mathbb{P}\left(X_{j}=\cdots=X_{j-m_{j}}=0\right) \\
& =\sum_{i=m_{j}}^{\infty} \sum_{z_{1}^{f(i)} \in A^{f(i)}} p\left(z_{1}^{f(i)} 10_{i}\right) \\
& =\sum_{i=m_{j}}^{\infty} \sum_{z_{1}^{f(i)} \in A^{f(i)}} p\left(0 \mid 0_{i-1} 1 z_{1}^{f(i-1)}\right) \cdots p\left(0 \mid 01 z_{1}^{f(1)}\right) p(0 \mid 1) p(1) \\
& \leq P(1) \sum_{i=m_{j}} \sum_{z_{1}^{f(i)} \in A^{f(i)}}(1-\delta)^{i}=P(1) \sum_{i=m_{j}}^{\infty}(1-\delta)^{i} 2^{f(i)} \\
& \leq P(1)\left(\sum_{i \in L}(1-\delta)^{i} 2^{i}+\sum_{i \in G}(1-\delta)^{f(i)} 2^{f(i)}\right)
\end{aligned}
$$

onde $L=\left\{i \geq m_{j}: f(i)<i\right\}$ e $G=\left\{i \geq m_{j}: f(i) \geq i\right\}$. Desta maneira

$$
\begin{aligned}
\mathbb{P}\left(l\left(X_{1}^{j}\right)>j-(n-k(n))\right) & \leq P(1)\left(\sum_{i \geq m_{j}}(2(1-\delta))^{i}+\sum_{i \geq m_{j}}(2(1-\delta))^{f(i)}\right) \\
& =P(1)\left(\frac{(2(1-\delta))^{m_{j}}}{1-2(1-\delta)}+\sum_{i \geq m_{j}}(2(1-\delta))^{f(i)}\right)
\end{aligned}
$$

desde que $\delta>1 / 2$. Assim

$$
\begin{aligned}
\mathbb{P}\left(\bigcup_{i=1}^{M_{n-k(n)}} t_{i} \notin \mathcal{S}\right) & \leq(n-k(n)) P(1) \sum_{j=n+1}^{\infty}\left(\frac{(2(1-\delta))^{m_{j}}}{1-2(1-\delta)}+\sum_{i \geq m_{j}}(2(1-\delta))^{f(i)}\right) \\
& =(n-k(n)) P(1)\left(\frac{1}{1-2(1-\delta)} \sum_{j=n+1}^{\infty}(2(1-\delta))^{m_{j}}+\sum_{j=n+1}^{\infty} \sum_{i \geq m_{j}}(2(1-\delta))^{f(i)}\right) \\
& =(n-k(n)) P(1)\left(\frac{1}{1-2(1-\delta)} \sum_{j=n+1}^{\infty}(2(1-\delta))^{m_{j}}+\sum_{j=1}^{\infty} j(2(1-\delta))^{f\left(m_{n+j}\right)}\right) \\
\rightarrow 0 &
\end{aligned}
$$


quando $n \rightarrow \infty$, desde que $2(1-\delta))^{f\left(m_{n}\right)}=o\left(\frac{1}{n-k(n)}\right)$ e $\sum_{j=n+1}^{\infty}(2(1-\delta))^{m_{j}}=o\left(\frac{1}{n-k(n)}\right)$.

Vamos agora considerar o caso em que temos um amostra $X_{1}, \ldots, X_{n}$ de $(\tau, p)$, com $\tau$ desconhecida. Neste caso, vamos usar o algoritmo do contexto para estimar a árvore de contextos associada à amostra, a qual chamaremos $\widehat{\tau}_{n}$ e então podemos usar o mesmo algoritmo descrito para o caso onde $\tau$ era conhecida, sendo que o passo 0 se torna:

Passo 0 - Fixe $\omega \in \widehat{\tau}_{n}$.

No caso onde $\tau$ é conhecida, os contextos não dependem da amostra. No caso em que $\tau$ é desconhecida, para cada amostra $X_{1}, \ldots, X_{n}$ temos uma estimativa $\widehat{\tau}_{n}$ e esta estimativa pode sofrer alterações a medida que a amostra muda de tamanho.

Para um dado contexto $\omega \in \widehat{\tau}_{n}$, teremos os pontos $t_{1}\left(X_{1}^{n}, \widehat{\tau}_{n}\right), \ldots, t_{M_{n}}\left(X_{1}^{n}, \widehat{\tau}_{n}\right)$ e com base nesses pontos, podemos dividir a amostra em blocos independentes $B_{0}=B_{0}\left(X_{1}^{n}, \widehat{\tau}_{n}\right), B_{1}=$ $B_{1}\left(X_{1}^{n}, \widehat{\tau}_{n}\right), \ldots, B_{M_{n}}=B_{M_{n}}\left(X_{1}^{n}, \widehat{\tau}_{n}\right)$, onde $B_{0}=\left[X_{1}, \ldots, X_{t_{1}-1}\right], B_{1}=\left[X_{t_{1}}, \cdots, X_{t_{2}-1}\right], \ldots$, $B_{M_{n}-1}=\left[X_{t_{M_{n}-1}}, \cdots, X_{t_{M_{n}}-1}\right]$ e $B_{M_{n}}=\left[X_{t_{M_{n}}}, \cdots, X_{n}\right]$. Para este caso em que não conhecemos a árvore de contextos de onde provém a amostra, apresentaremos um limitante superior para a probabilidade de que um determinado bloco $B_{i}$ não pertença ao conjunto $\mathcal{B}$ dos blocos de $X_{1}, X_{2}, \ldots$

Teorema 4.3 Seja $X_{1}, \ldots, X_{n}$ uma amostra de $(\tau, p)$, onde $\tau$ é desconhecida. Considerando $B_{0}\left(X_{1}^{n}, \widehat{\tau}_{n}\right), \ldots, B_{M_{n}}\left(X_{1}^{n}, \widehat{\tau}_{n}\right)$ os blocos de separação associados a esta amostra, então

$$
\mathbb{P}\left(\bigcup_{i=0}^{M_{n}} B_{i}\left(X_{1}^{n}, \tau_{n}\right) \notin \mathcal{B}\right) \rightarrow 0
$$

\section{Prova:}


Temos que

$$
\mathbb{P}\left(\bigcup_{i=1}^{M_{n}}\left\{B_{i} \notin \mathcal{B}\right\}\right) \leq \sum_{i=1}^{M_{n}-1} \mathbb{P}\left(B_{i} \notin \mathcal{B}\right) .
$$

Mas $B_{i} \notin \mathcal{B}$ acontece se:

- $t_{i} \notin \tau$ ou

- $t_{i} \in \tau$ e $t_{i} \notin \mathcal{S}$ ou

- $t_{i+1} \notin \tau$ ou

- $t_{i+1} \in \tau$ e $t_{i+1} \notin \mathcal{S}$.

Assim,

$$
\mathbb{P}\left(B_{i} \notin \mathcal{B}\right) \leq \mathbb{P}\left(t_{i} \notin \tau\right)+\mathbb{P}\left(t_{i} \in \tau, t_{i} \notin \mathcal{S}\right)+\mathbb{P}\left(t_{i+1} \notin \tau\right)+\mathbb{P}\left(t_{i+1} \in \tau, t_{i+1} \notin \mathcal{S}\right)
$$

Mas, podemos ver em Duarte et al. (2006) que $\widehat{\tau}_{n} \rightarrow \tau$ em probabilidade e portanto $\mathbb{P}\left(t_{i} \notin \tau\right) \rightarrow 0$ e $\mathbb{P}\left(t_{i+1} \notin \tau\right) \rightarrow 0$. E pelos teoremas que demonstramos para o caso $\tau$ conhecida, concluímos que

$$
\mathbb{P}\left(\bigcup_{i=0}^{M_{n}} B_{i}\left(X_{1}^{n}, \tau_{n}\right) \notin \mathcal{B}\right) \rightarrow 0 .
$$




\section{Referências Bibliográficas}

Bertail, P. \& Clémençon, S. (2006). Regenerative block-bootstrap for Markov chains. Bernoulli Journal 12(4), 689-712.

Bühlmann, P. \& Wyner, A. J. (1999). Variable length Markov chains. The Annals of Statistics 27(2), 480-513.

Csiszár, I. \& TAlata, Z. (2006). Context tree estimation for not necessarily finite memory processes, via BIC and MDL. IEEE Transactions on Information Theory 52(3), 1007-1016.

Duarte, D. (2003). Aproximações Markovianas e Reamostragem para Cadeias de Ordem Infinita com Aplicação à Lingüistica. Ph.D. thesis, IME-USP.

Duarte, D., Galves, A. \& Garcia, N. L. (2006). Markov approximation and consistent estimation of unbounded probabilistic suffix trees. Unpublished.

Fernández, R. \& Galves, A. (2002). Markov approximations of chains of infinite order. Bull. Braz. Math. Soc. 33, 295-306.

Fernández, R., Ferrari, P. A. \& Galves, A. (2001). Coupling, renewal and perfect simulation of chains of infinite order. Vth Brazilian School of Probability. 
FERrari, F. \& Wyner, A. (2003). Estimation of general stationary processes by variable length Markov chains. Scandinavian Journal of Statistics 30(3), 459-480. URL http://www.blackwell-synergy.com/doi/abs/10.1111/1467-9469.00342.

Ferrari, P. A. \& Galves, A. (1997). Acoplamento em processos Estocásticos. IMPA, Rio de Janeiro, Brasil.

Khuri, A. I. (2003). Advanced Calculus with Applications in Statistics. Wiley, second ed.

LALley, S. (2000). Regeneration in one-dimensional Gibbs states and chains with complete connections. Resenhas do Instituto de Matemática e Estatística da Universidade de São Paulo 4(3), 249-281.

Mächler, M. \& Bühlmann, P. (2002). Variable length Markov chains: Methodology, computing and software. Tech. rep., Eidgenössische Technische Hochschule (ETH).

Rissanen, J. (1983). A universal data compression system. IEEE Transactions on Information Theory IT-29, 656-664. 